\title{
Oldenlandia diffusa Promotes Antiproliferative and Apoptotic Effects in a Rat Hepatocellular Carcinoma with Liver Cirrhosis
}

\author{
Yun-Young Sunwoo, ${ }^{1}$ Jin-Hee Lee, ${ }^{2}$ Ho Yong Jung, ${ }^{3}$ Yu Jin Jung, \\ Moon-Seo Park, ${ }^{3}$ Yong-An Chung, ${ }^{3,5}$ Lee-So Maeng, ${ }^{3}$ Young-Min Han, ${ }^{3}$ \\ Hak Soo Shin, ${ }^{3}$ Jisoo Lee, ${ }^{6}$ and Sang In Park ${ }^{3}$ \\ ${ }^{1}$ Comprehensive Hospital for Advanced Cancer, International St. Mary's Hospital, College of Medicine, \\ The Catholic Kwandong University of Korea, Incheon 404-834, Republic of Korea \\ ${ }^{2}$ Department of Pharmacy, College of Pharmacy, Seoul National University, Seoul 151-742, Republic of Korea \\ ${ }^{3}$ Institute of Catholic Integrative Medicine (ICIM), Incheon St. Mary's Hospital, The Catholic University of Korea, \\ Incheon 403-720, Republic of Korea \\ ${ }^{4}$ EIT/LOFUS Research Center, International St. Mary's Hospital, College of Medicine, \\ The Catholic Kwandong University of Korea, Incheon 404-834, Republic of Korea \\ ${ }^{5}$ Department of Radiology, Incheon St. Mary's Hospital, The Catholic University of Korea, Incheon 403-720, Republic of Korea \\ ${ }^{6}$ Department of Internal Medicine, University of Massachusetts Medical School, Worcester, MA 01655, USA
}

Correspondence should be addressed to Lee-So Maeng; mis1004@olmh.cuk.ac.kr and Sang In Park; parksi07@catholic.ac.kr

Received 9 December 2014; Revised 9 February 2015; Accepted 11 February 2015

Academic Editor: Jairo Kennup Bastos

\begin{abstract}
Copyright (C) 2015 Yun-Young Sunwoo et al. This is an open access article distributed under the Creative Commons Attribution License, which permits unrestricted use, distribution, and reproduction in any medium, provided the original work is properly cited.
\end{abstract}

Oldenlandia diffusa (OD) is commonly used with various diseases such as cancer, arthritis, and autoimmune disease. Liver cirrhosis is a predominant risk factor for hepatocellular carcinoma (HCC). Here, we show that the therapeutic effect of OD, which was investigated both in vitro and chemically, induced HCC model. OD significantly enhanced apoptosis and antiproliferative activity and reduced migration ability of HCC cells. In vivo, OD was treated twice a day for 28 days after confirmed HCC model through 2- $\left[{ }^{18} \mathrm{~F}\right]$-fluoro-2-deoxy-D-glucose $\left({ }^{18} \mathrm{~F}-\mathrm{FDG}\right)$ imaging. The survival in OD treated groups was shown to have a greater therapeutic effect than the control group. 28 days after OD treatment, OD treated groups resulted in a significant reduction in tumor number, size, ${ }^{18}$ F-FDG uptake, and serum levels such as alanine transaminase, aspartate transaminase, and alkaline phosphate compared to the control group. Also, proliferated cells in tumor sites by OD were reduced compared to the control group. Furthermore, several rats in OD treated group survived over 60 days and liver morphology of these rats showed the difference between tumor mass and normal tissue. These results suggest that OD may have antiproliferative activity, inhibition of metastasis, and apoptotic effects in chemically induced HCC model and can have the potential use for clinical application as anticancer drug of the herbal extract.

\section{Introduction}

Hepatocellular carcinoma (HCC) is the fifth most common cancer and the second leading cause of cancer death worldwide $[1,2]$. HCC can be involved from hepatitis B or hepatitis $\mathrm{C}$, cirrhosis due to alcohol consumption, liver disease such as aflatoxin toxicity, hormonal imbalance, and metabolic disease [3-5]. Various treatment options for HCC are available clinically, which include surgery, chemotherapy, and radiotherapy. However, the patients are at high risk in recurrence and the progressing mechanism of HCC including long-lasting inflammation in hepatocytes leading to cirrhosis has not been fully elucidated $[6,7]$.

A number of studies have reported the beneficial effect of herbal medicine in diverse diseases such as central nervous system and cancer. Also, many herbal drugs and prescriptions have in fact been used clinically for the treatment of cancer and autoimmune diseases. Several studies reported anticancer activity in vitro and in vivo using natural herb extract [8-10]. Recently, an extract isolated from medicinal 
herb such as Oldenlandia diffusa (OD) has intrigued interest because of its anticancer and chemoprevention effect on various cancer and normal cells $[11,12]$.

$\mathrm{OD}$ is a well-known medicinal plant used in Korean and Chinese herbal medicine for the treatment of hepatitis, tonsillitis, urethral infection, and malignant tumors of the liver and lung. A number of studies have reported multiple biological activities of the OD such as antitumor, chemopreventive, anti-inflammatory, antioxidant, and proapoptotic effect $[13,14]$. However, the mechanism behind the antitumor effect of OD is still largely unknown and has certainly not been evaluated in chemically induced liver cancer. OD is identified to include oleanolic acid (3b-hydroxyolean-12en-28-oic acid, OA), ursolic acid (3b-3-hydroxyurs-12-ene28-oic acid, UA), asperuloside (IG1), E-6-O-p-coumaroyl scandoside methyl ester (IG2), and E-6-O-p-coumaroyl scandoside methyl ester-10-methyl ether (IG3). OA has an isomer, UA, which is also a pentacyclic triterpenoid compound. $\mathrm{OA}$ and UA are distinguished by the position of methyl group between $\mathrm{C} 19$ and $\mathrm{C} 20$. It has also been reported that dexamethasone, which is a glucocorticoid hormone that has a structure similar to OA and UA. Although OA and UA have similar structure, OA has effects such that the inhibitory effect of OA on cytochrome P450 is stronger than UA [15]. Also, $\mathrm{OA}$ and UA exhibit significant antitumor effect and cytotoxic activity in many cancer cell lines such as liver cancer cells, gastric cancer cells, colon carcinoma cells, and fibrosarcoma cells $[11,16-20]$.

In this study, we investigated the ability of OD enhanced anticancer effect in apoptotic cell death, inhibition of proliferation, and migration of HCC cell lines. In addition, we were able to demonstrate OD enhanced antitumor effects in regulation of hepatic function, glucose metabolism, and metastasis in chemically induced liver cancer model.

\section{Materials and Methods}

2.1. Preparation of Oldenlandia diffusa (OD), Oleanolic Acid $(\mathrm{OA})$, and Ursolic Acid (UA). The herbal sample of OD Roxb was purchased from an herbal market in Gyeongdong. OD was extracted using reflux extraction equipment in hot water for an hour and concentrated using a rotary evaporator before lyophilization. OA and UA were purchased from SigmaAldrich (St. Louis, MO, USA). The compounds were dissolved in DMSO and the final concentration of DMSO in samples was $0.15 \%$.

2.2. Cell Culture. Liver cancer cell lines such as huh7 and hepG2 were obtained from the American Type Culture Collection (ATCC; Manassas, VA, USA, http://www.atcc.org). The cells were maintained in Dulbecco's Modified Eagle's Medium (DMEM; Hyclone, Logan, UT, USA). The huh7 and hepG2 cells were supplemented with antibiotics and $10 \%$ of fetal bovine serum (FBS; HyClone). Cells were incubated at $37^{\circ} \mathrm{C}$ in a humidified atmosphere containing $5 \% \mathrm{CO}_{2}$.

2.3. Cell Viability Assay. Cell viability was measured using a Cell Counting Kit-8 (CCK-8; Dojindo Laboratories) allowing sensitive colorimetric assay by using highly water-soluble tetrazolium salt that is reduced by dehydrogenases in living cells to give a colored product (formazan). The cells were seeded in $96-\left(5 \times 10^{3}\right.$ cells/well $)$ or 24 -well plates $(2 \times$ $10^{4}$ cells/well) in medium containing $10 \%$ FBS and were incubated for $24 \mathrm{~h}$. Subsequently, cells were treated with medium containing OA $(0-100 \mu \mathrm{g} / \mathrm{mL})$, UA $(0-100 \mu \mathrm{g} / \mathrm{mL})$, and $\mathrm{OD}(0-500 \mathrm{mg} / \mathrm{mL})$ at $37^{\circ} \mathrm{C}$ for $24 \mathrm{~h}$. The untreated cells in medium containing $0.15 \%$ DMSO were used as $100 \%$ viability controls. The cells with CCK- 8 were incubated for $2 \mathrm{~h}$ and were then measured at $450 \mathrm{~nm}$ with a spectrophotometer (Molecular Devices, Sunnyvale, CA, USA).

2.4. Scratch-Induced Migration Assay. To investigate the effect of OD, OA, and UA on the migration of HCC cells, a scratch-induced migration assay was performed. The cells were seeded in 6-well plates $\left(1 \times 10^{5}\right.$ cells/well $)$ and maintained in DMEM with 10\% FBS. The monolayer cells were scraped by using the tip of a $200 \mu \mathrm{L}$ micropipette, creating linear streaks $2 \mathrm{~mm}$ apart. Cells were washed twice with medium to remove nonadherent cells and were further incubated in fresh medium with $10 \%$ FBS containing OD $(200 \mathrm{mg} / \mathrm{mL}), \mathrm{OA}(100 \mu \mathrm{g} / \mathrm{mL})$, and UA $(50 \mu \mathrm{g} / \mathrm{mL})$ for $48 \mathrm{~h}$. Wound closure or cell migration was photographed at 0 , 24 , and $48 \mathrm{~h}$ after wounding, using an inverted microscope equipped with digital camera (Nikon, Tokyo, Japan). The images were analyzed into the Meta-Morph imaging software (Molecular Devices Inc., Downingtown, PA, USA). For statistical analysis, at least three independent scratch wound experiments were used for calculations.

2.5. Real Time Polymerase Chain Reaction Analysis. Total RNA was extracted from cancer cells using RNeasy Mini Kit (Qiagen, Valencia, CA). cDNA was synthesized with total RNA and oligo(dT) primer (Invitrogen, Carlsbad, CA) using Omniscript RT kit (Qiagen) for real time polymerase chain reaction (RT-PCR). The cDNA samples were diluted 20-fold with water and were used as templates for RT-PCR with $2 \times$ SYBR Green Master Mix and oligonucleotide primers. Quantitative RT-PCR was performed using the MyiQ Single-Color RT-PCR Detection System (TaKaRa, Shiga, Japan). Details of the primers are described in the Supporting Information (Supplementary Table 1) (see Supplementary Materials available online at http://dx.doi.org/10.1155/2015/501508). RTPCR was conducted as described previously [21]. Standard PCR conditions were $95^{\circ} \mathrm{C}(5 \mathrm{~min})$ and then 40 cycles of $94^{\circ} \mathrm{C}(10 \mathrm{~s}), 60^{\circ} \mathrm{C}(20 \mathrm{~s})$, and $72^{\circ} \mathrm{C}(15 \mathrm{~s})$, followed by the standard denaturation curve with $0.5^{\circ} \mathrm{C}$ increment for 70 cycles. The $\Delta \mathrm{Ct}$ method was used to calculate the relative levels of expression. The threshold cycle $(\mathrm{Ct})$ value from triplicate measurements was calculated for the expression of the target gene and normalized to that of GAPDH.

2.6. Animal Model. The experimental protocols used in this study were designed according to the animal experimental guidelines established by the Institutional Animal Care and Use Committee of Catholic University Medical School. The HCC model procedure was performed as described previously [22, 23]. To induce HCC model, male Sprague-Dawley (180-200 g) rats received intraperitoneal injections of DEN 
(50 mg/kg; Sigma, St. Louis, MO, USA) once a week for 16 weeks. We confirmed successful development of HCC through PET/CT scan at the last DEN injection.

2.7. Experimental Groups and Therapeutic Efficacy Study. Animals were assigned randomly to one of the following three major groups: (1) one group of rats was treated with distilled water as control group $(n=9)$, (2) the second group was fed with OD $(100 \mathrm{mg} / \mathrm{kg} ; n=9)$, and (3) the third group was fed with OD $(200 \mathrm{mg} / \mathrm{kg} ; n=9)$. To assess the survival of the OD treated animals, rats were randomized ( $n=9$, for each group) after last DEN injection and were treated with OD twice a day for 28 days. We recorded starting from day 0 after OD treatment. Survival was followed for a maximum of 60 days.

2.8. PET/CT Imaging and Data Analysis. PET/CT imaging and data analysis were performed as previously reported $[24,25]$. Images were taken at 0 and 28 days after the OD treatment with a PET scanner using a General Electric Discovery STE (Waukesha, WI, USA). The rats were deeply anesthetized with ketamine and xyzine and ${ }^{18}$ F-FDG $(1.1 \pm$ $0.04 \mathrm{mCi}$ ) was injected intravenously into the caudal vein. After $30 \mathrm{~min}$, the rats were taken using a PET/CT scanner and body temperature was kept at $37^{\circ} \mathrm{C}$ with a heating pad on the scanner bed.

To assess the changes of ${ }^{18} \mathrm{~F}$-FDG uptake, regions of interest (ROIs) in the lesioned areas were identified in images of the coronal and horizontal sections. Each ROI value was defined on the coronal tomograms that showed the highest uptake to be in the middle of the tumor and calculated as the averaged $\mathrm{nCi} / \mathrm{cc}$ after calibration of both liver of insulated areas and muscle in the same images. We expressed the standard uptake value (SUV) in ROIs values.

2.9. Liver Function Tests. To test liver function, we examined the serum levels of alkaline phosphatase (ALP; Siemens), aspartate transaminase (AST; Siemens), and alanine transaminase (ALT; Siemens, USA) 28 days after OD treatment. Briefly, the blood samples were collected before sacrifice and centrifuged at $3000 \mathrm{rpm}$ for $20 \mathrm{~min}$. The supernatant was analyzed to quantify the concentration in strict accordance with the manufacturer's protocols.

2.10. Macroscopic and Microscopic Observation. Four weeks after the last DEN injection, all group rats $(n=5$, for each group) were deeply anesthetized with $15 \%$ urethane and sacrificed by decapitation. The whole liver tissue was sliced into $2 \mathrm{~mm}$ thickness. The tumors were counted by macroscopic examination of the liver through two independent investigators according to the criteria.

2.11. Histological Examination. 28 days after OD treatment, animals from each group were sacrificed for histological examination. The tissue was cut coronally at a thickness of $4 \mu \mathrm{m}$ from each rat for a total of three blocks. The paraffin sections were dewaxed in histoclear and rehydrated through a grade alcohol series. The slides were incubated with primary antibody against mouse antiproliferating cell nuclear antigen (PCNA; Millipore, Billerica, MA, USA) at $4^{\circ} \mathrm{C}$ overnight.
Subsequently, slides were incubated in Alexa 546-conjugated goat anti-mouse IgM (Molecular probe, Eugene, Oregon, USA). The slides were counterstained with $4^{\prime}, 6$-diamidino2-phenylindole (DAPI; Sigma-Aldrich) and detected using a Zeiss LSM510 confocal microscope (Carl Zeiss, Jena, Germany).

2.12. Statistical Analysis. All data are presented as mean values \pm standard deviation from at least 3 independent experiments. Statistical differences between different test conditions were determined using Student's $t$-test. Probability values less than 0.05 were considered statistically significant. Also, the statistical analysis of survival was carried out using a log-rank test.

\section{Result}

3.1. Anticancer Effect of Oldenlandia diffusa (OD) in Hepatocellular Carcinoma (HCC) Cells. First, we determined the antitumor effect of OD including cytotoxicity and growth inhibitory effect in hepatocellular carcinoma cells such as huh7 and hepG2. OD treatment exhibited a cytotoxic effect in a dose-dependent manner (Figure 1(a)). Based on these results, we chose OD concentration of $200 \mathrm{mg} / \mathrm{mL}$ for the following in vitro experiments. We treated HCC cells with OD for $72 \mathrm{~h}$ and assessed the number of viable cells. We showed that OD has time dependent growth inhibitory effect (Figure 1(b)). Also, we observed caspase-3 and ki67 through immunostaining (Figure 1(c)). In OD treatment, the activity of ki67-positive cell of HCC cells was decreased and the activation of caspase- 3 was significantly increased compared to the control (Figure 1(d)). We questioned what components of OD have antitumor effects in HCC cells. Numerous studies have reported that the principal component in OD including $\mathrm{OA}$ and UA could have antitumor effects. We showed that OA and UA have antitumor effects in HCC cells (Supplementary Figure 1). These results suggest that $\mathrm{OD}$ has an antitumor effect via the antiproliferation activity and apoptosis of $\mathrm{OA}$ and UA in HCC cells.

3.2. Suppression of HCC Cell Migration by OD. To examine the effect of OD on cell migration, we performed the scratch wound healing assay using the huh7 and hepG2 cells. All wound healing images are at the same magnification and time after OD treatment. At $48 \mathrm{~h}$, the wound of FBS treatment was healed approximately $65 \% \pm 13 \%$ in huh7 and $53.4 \%$ $\pm 11 \%$ in hpeG2 cells from the positive-control cells. OD significantly reduced cell motility in both huh7 (OD: 36.8\% $\pm 6 \%$ ) and hepG2 (OD: $37.4 \% \pm 4 \%$ ) compared to the FBS treatment (Figure 2). Furthermore, we showed that OA and UA treatment reduced wound healing (Supplementary Figure 2). We also measured mRNA for migration related receptors such as CXCR1, CXCR2, and CXCR4 in HCC cells by OD, $\mathrm{OA}$, and UA. The expression of migration related receptor was significantly decreased by OD, OA, and UA compared to the FBS treatment (Figure 2(c); Supplementary Figure 2). These results suggested that the OD could inhibit the migration in HCC cells through inhibition of migration receptors by OA and UA. 


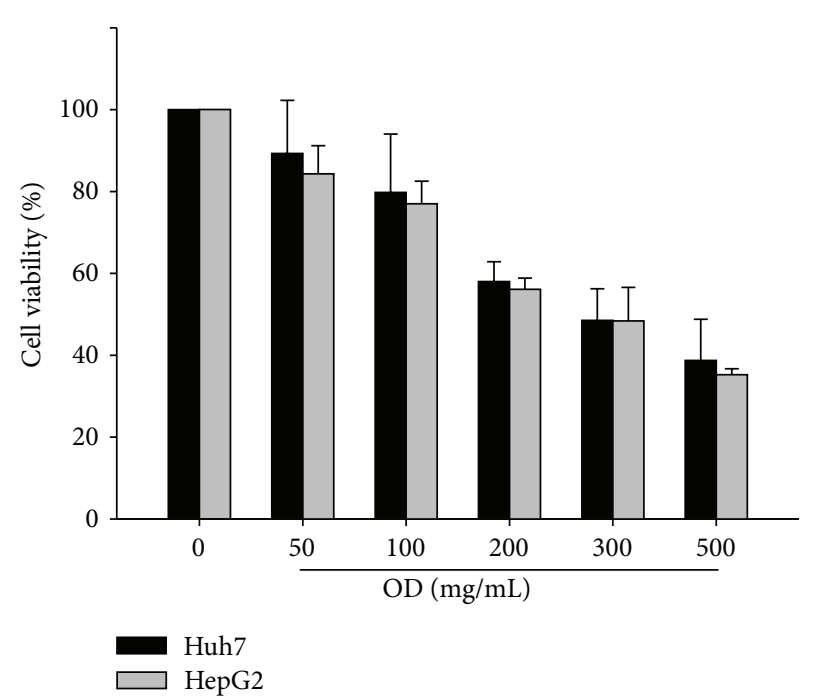

(a)

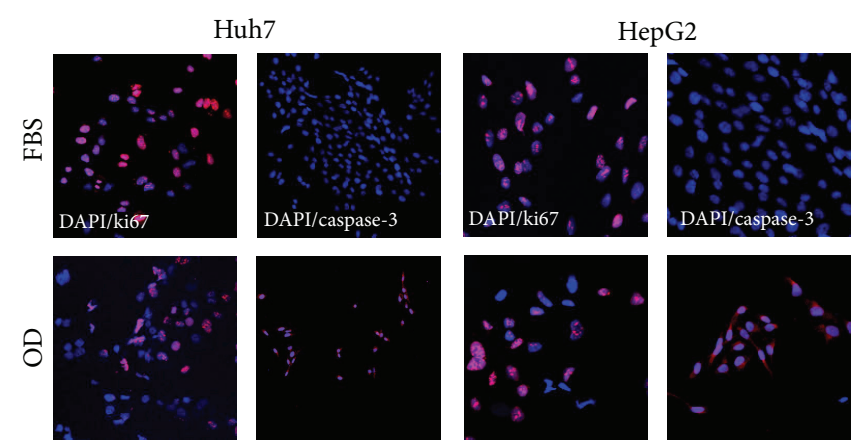

(c)

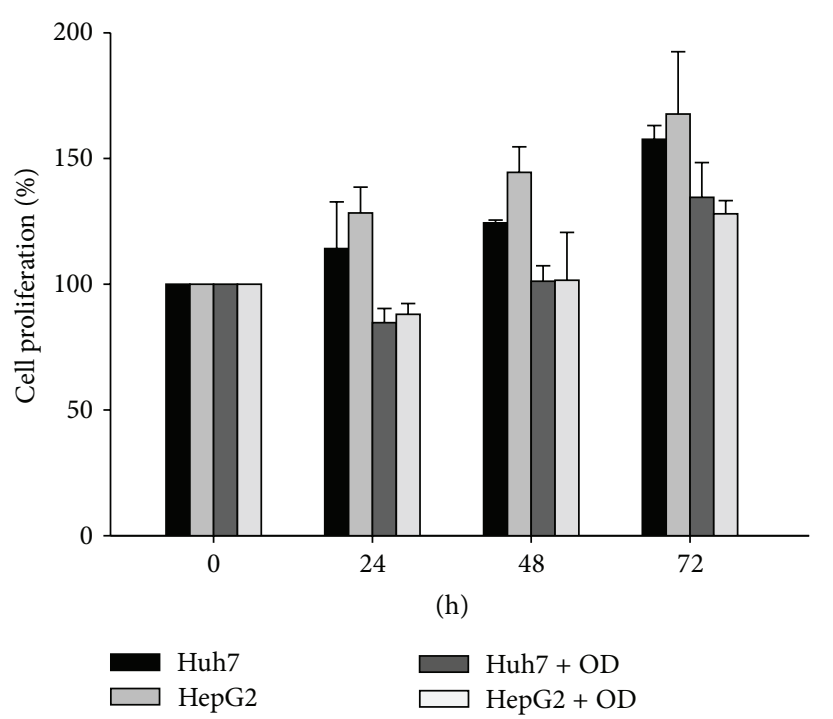

(b)

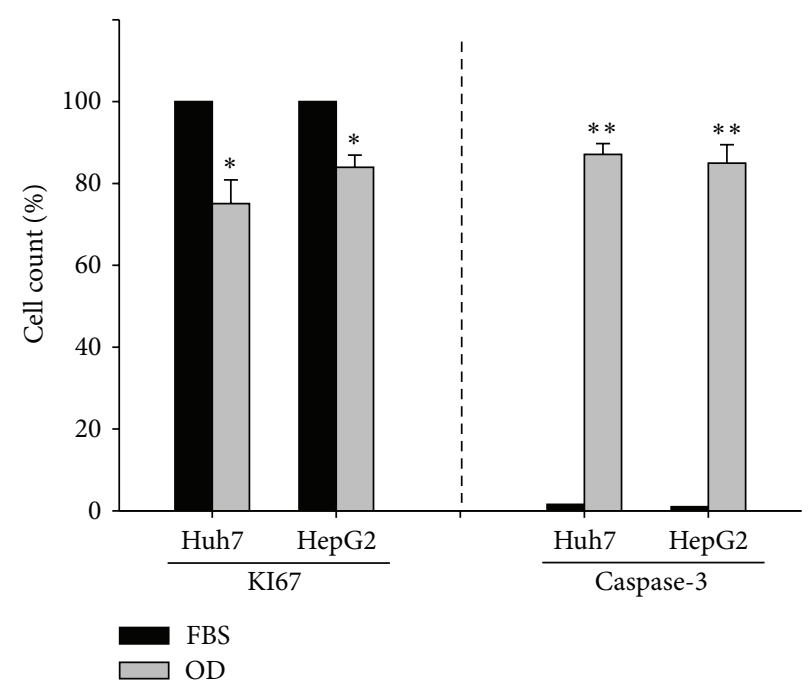

(d)

FIgURE 1: The antitumor effect on hepatocellular carcinoma (HCC) cell lines treated with Oldenlandia diffusa (OD). The cytotoxic effect and antiproliferative activity were measured by CCK-8 cytotoxicity assay and immunoreactive cells such as Ki67 and caspase-3. The cytotoxicity effect on HCC cell lines treated with the indicated concentrations of OD (0-500 mg/mL) for $24 \mathrm{~h}(\mathrm{a})$. Also, we measured antiproliferative activity of OD (200 mg/mL) against HCC cells for $72 \mathrm{~h}$ (b). Ki67 and caspase-3 staining were performed after $24 \mathrm{~h}$ in OD (200 mg/mL) ((c), (d); scale bars, $100 \mu \mathrm{m})$. OD treatment in huh7 and hepG2 cells reduced ki67-positive cells and increased caspase-3 immunoreactivity. Columns, mean; bar, SE. Student's $t$-test, ${ }^{*} P<0.05$ and ${ }^{* *} P<0.01$.

3.3. Survival and Uptake of ${ }^{18}$ F-FDG in HCC Model by OD. Before treating OD, we confirmed HCC model using the $\mathrm{PET} / \mathrm{CT}$ imaging. We recorded starting from day 0 before $\mathrm{OD}$ treatment and observed the survival rate of rats. $50 \%$ of rats in control group died between 20 days and 30 days (Figure 3(b)). The last rat of control group was dead after 36 days. However, the rats of OD treated group (100 and $200 \mathrm{mg} / \mathrm{kg}$ ) survived more than $40 \%$ (Figures $3(\mathrm{a})$ and $3(\mathrm{~b})$ ). Also, $30 \%$ of rats in OD treated group $(200 \mathrm{mg} / \mathrm{kg})$ survived more than 50 days. PET/CT imaging was performed on day 0 and day $28 .{ }^{18} \mathrm{~F}$ FDG has long been demonstrated to be a marker of glucose metabolism in cirrhosis and tumor. The specific areas of liver identified with increased uptake of ${ }^{18} \mathrm{~F}-\mathrm{FDG}$ in PET imaging (Figure $3(\mathrm{c})$ ) and the quantitative image analysis of ${ }^{18} \mathrm{~F}$-FDG revealed the SUV (Figure 3(d)). The SUV value of liver in OD treated group (100 and $200 \mathrm{mg} / \mathrm{kg}$ ) was lower in certain areas compared to the control group (control: $132.0 \pm 11.7$; OD $100 \mathrm{mg} / \mathrm{kg}: 112.5 \pm 7.2$; OD $200 \mathrm{mg} / \mathrm{kg}: 108.8 \pm 5.7)$.

3.4. Reduction for Number of Tumors and Levels of ALT, AST, and $A L P$ in Serum by OD. We investigated whether OD could change hepatic function related factors including ALT, AST, and ALP on serum level in DEN-induced HCC model. We collected serum 28 days after OD treatment and analyzed 

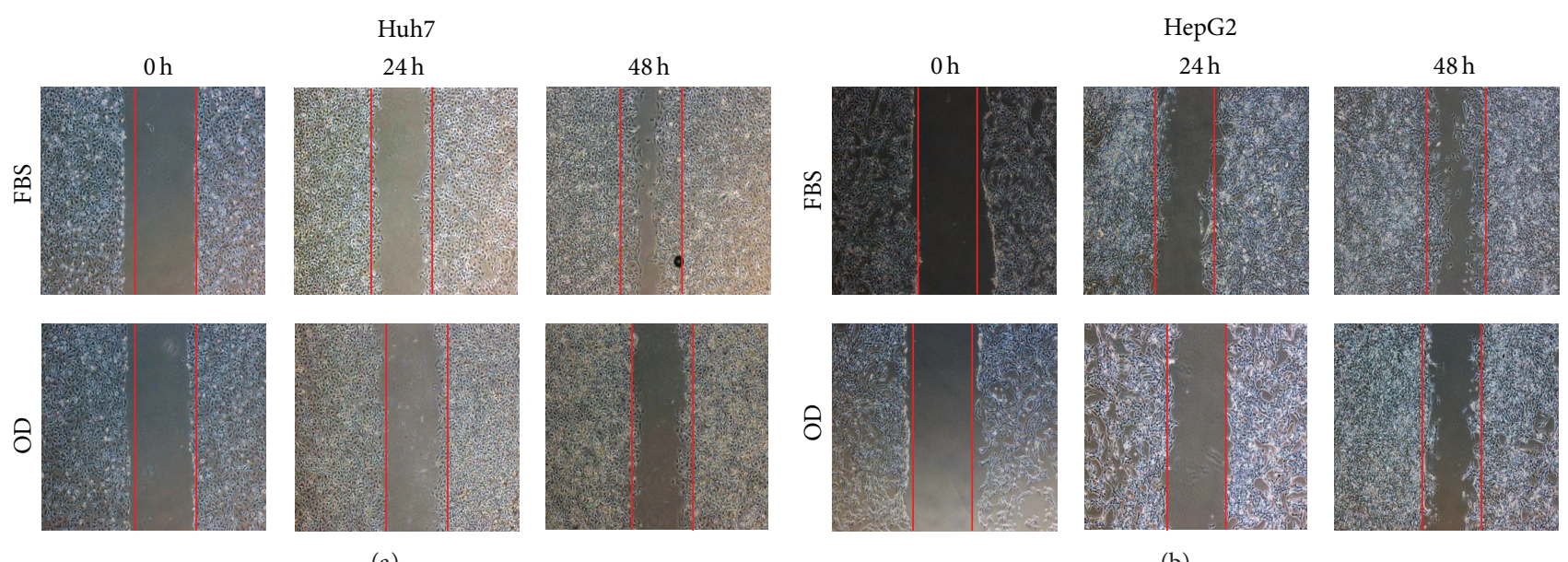

(a)

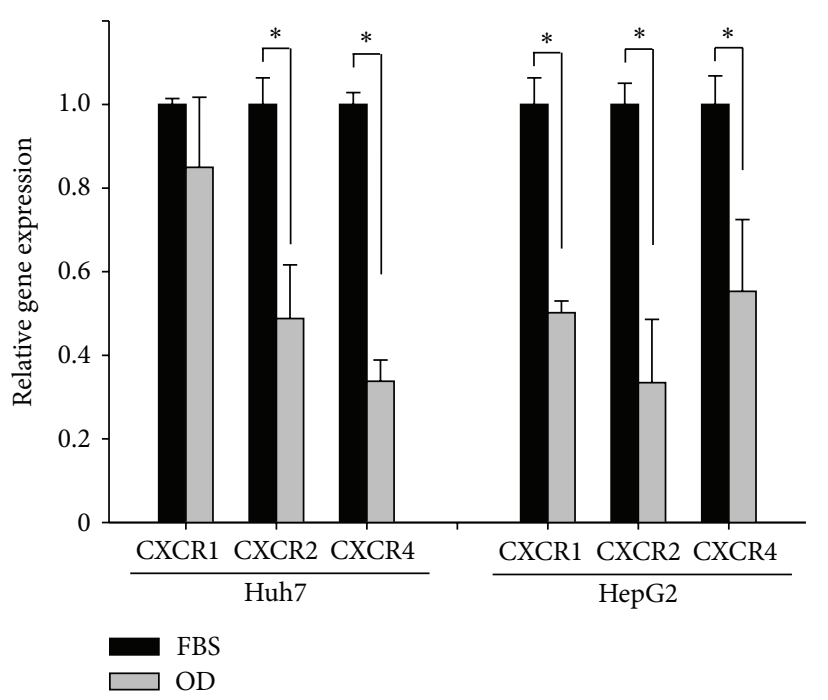

(c)

(d)

FIGURE 2: The migration effect of HCC cells by OD. Effect of OD on cell migration by scratch wound migration assay ((a), (b), and (c)). 10\% FBS containing DMEM was used as an experimental control. At $48 \mathrm{~h}$ after scratching, cell migration is suppressed significantly in huh7 and hepG2 by OD $(200 \mathrm{mg} / \mathrm{mL})(\mathrm{c})$. OD influences the expression of migration related receptor including CXCR1, CXCR2, and CXCR4 (d) for 24 h. Columns, mean; bar, SE. Student's $t$-test, ${ }^{*} P<0.05$ and ${ }^{* *} P<0.01$.

component of related liver function. Also, a comparison was made by categorizing the tumor number by tumor size and measured the liver/body ratio 28 days after OD treatment. The serum ALP, AST, and ALT levels in OD treated group (100 and $200 \mathrm{mg} / \mathrm{kg}$ ) were significantly lower compared to the control group (Figure 4(a)). Also, the size distribution of nodules and the liver/body ratio in OD treated group (100 and $200 \mathrm{mg} / \mathrm{kg}$ ) were significantly reduced compared to the control group (Figures 4(b) and 4(c)). These results suggest that OD may regulate the development and metastasis in tumors by enhancing hepatic function.

\subsection{Suppression of Tumor Cell Proliferation and Metastasis} by $O D$. To investigate the inhibitory effect of $\mathrm{OD}$ on the proliferation in tumor cells, we performed immunostaining in PCNA, which is a cell proliferation marker. In OD group, PCNA-positive cells were significantly decreased compared to the control group (Figure 5). Also, we observed the hepatic morphology of rats more than 60 days after OD treatment. 60 days after OD treatment $(200 \mathrm{mg} / \mathrm{kg})$, the large tumor was observed in two sites (Figure 6(a)). Except for tumor site, the rest of the liver tissue was similar in morphology to the normal liver. We think that this is very interesting result because of numerous developments of multinodular HCC and cirrhosis in chemically induced HCC model. Also, the percentage of liver/body weight ratios in rats 28 days and 60 days after OD treatment $(200 \mathrm{mg} / \mathrm{kg}$ ) was not significantly different (Figures 4(c) and 6(b)). Furthermore, we demonstrated a significant tumor growth including malignant nodules over $3 \mathrm{~mm}$ of a diameter, cirrhosis, and necrosis using the H\&E staining at 28 days in control group (Figures 6(c)-6(f)). 60 days after OD treatment $(200 \mathrm{mg} / \mathrm{kg})$, a boundary line between the large tumor and normal liver cells was observed (Figures $6(\mathrm{~g})-6(\mathrm{f})$ and $6(\mathrm{k})-6(\mathrm{~m}))$, as well as normal hepatocellular 

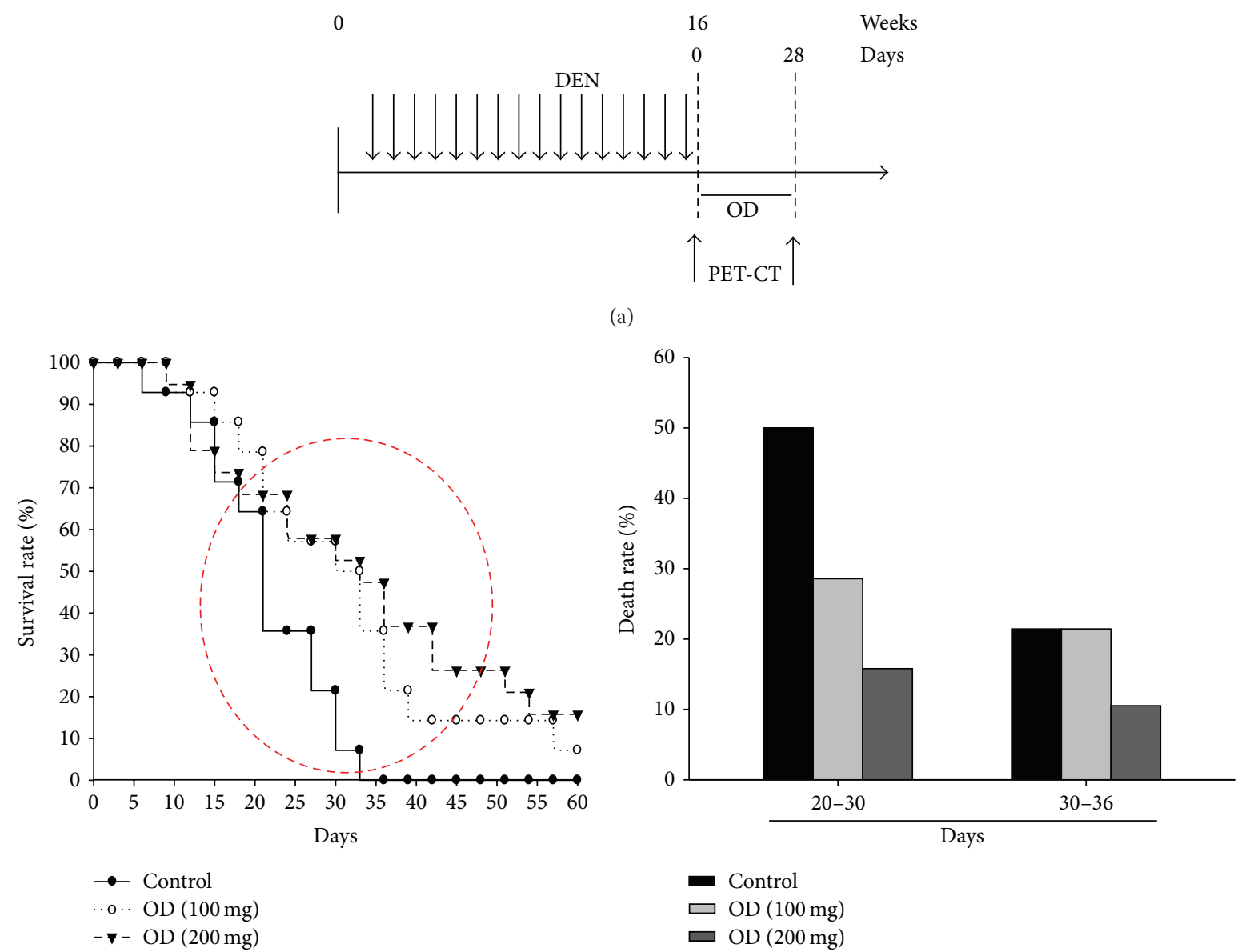

(b)
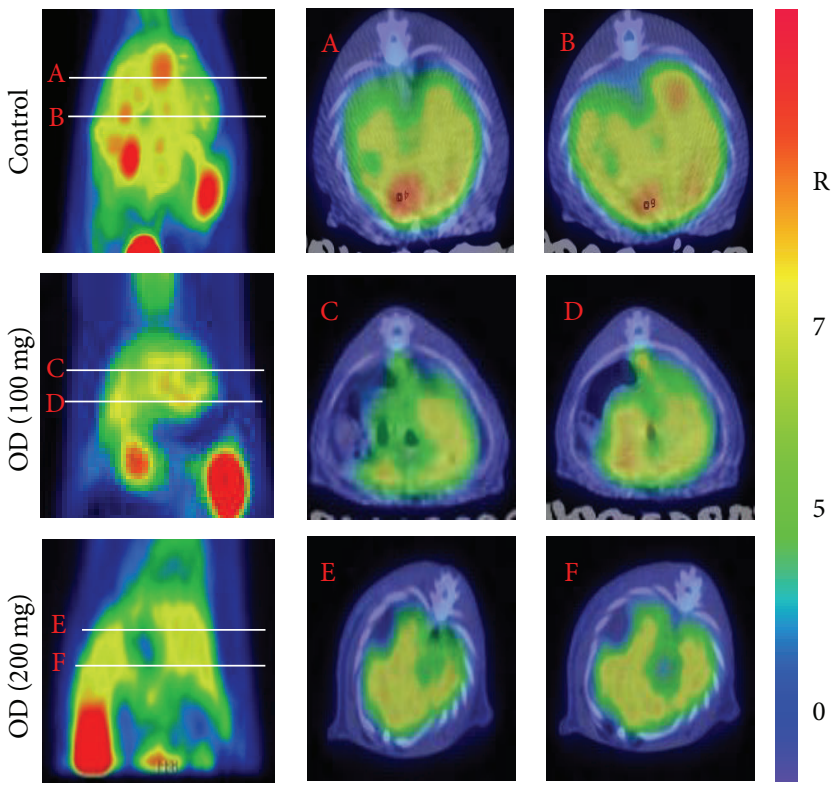

(d)

(c)

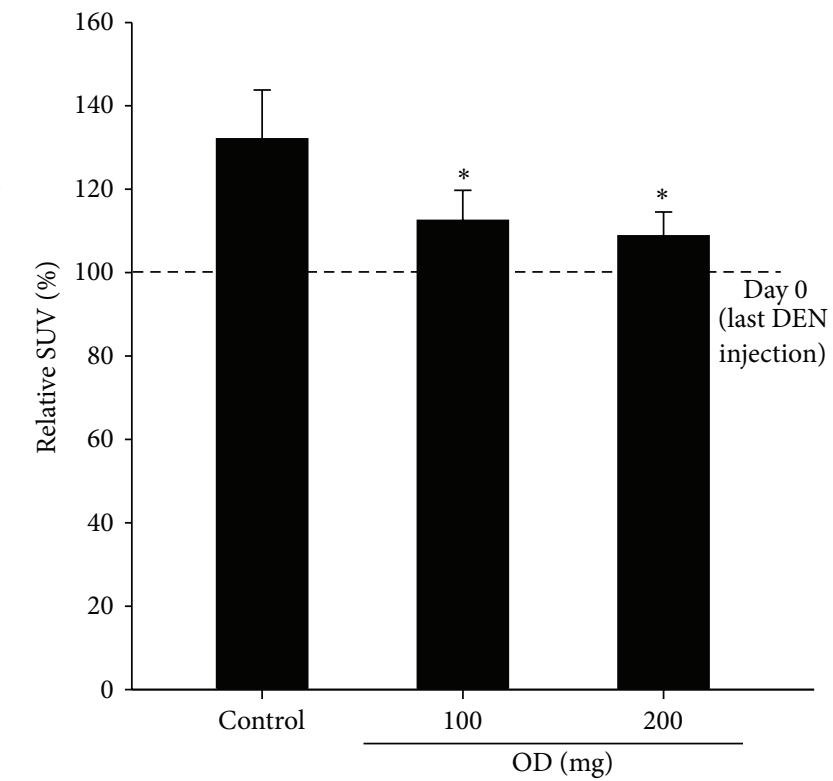

(e)

FIGURE 3: The therapeutic effects of OD for survival and glucose metabolism after HCC. Experimental scheme to test the effect of OD on the survival or ${ }^{18}$ F-FDG uptake in HCC model (a). The survival of HCC was analyzed by a log-rank test based on the Kaplan-Meier method (b). We recorded starting from day 0 after the last DEN injection. We record death rate between 20 days and 36 days (c). ${ }^{18}$ F-FDG PET was scanned at day 0 and 28 days after OD treatment. At 28 days after OD treatment, liver of rats in control group was significantly enhanced uptake of ${ }^{18}$ F-FDG into generated HCC and showed multinodular HCC. However, uptake of ${ }^{18} \mathrm{~F}$-FDG on rat's liver in OD (100 and $200 \mathrm{mg}$ ) treated group was decreased compared to the control group (d). Quantitative analysis of ${ }^{18} \mathrm{~F}$-FDG uptake analyzed at day 0 and 28 days after OD treatment (e). The relative SUV of OD treated group was decreased compared to the control group. Columns, mean; bar, SD. ${ }^{*} P<0.05$. 


\begin{tabular}{lccc}
\hline & ALP (U/L) & AST (U/L) & ALT (U/L) \\
\hline Normal & $150.6 \pm 30.2$ & $110.0 \pm 15$ & $42.0 \pm 3.0$ \\
Control & $215.6 \pm 18.4$ & $338.0 \pm 5.5$ & $174.3 \pm 56.9$ \\
OD $(100 \mathrm{mg})$ & $178.5 \pm 4.5$ & $347.0 \pm 5.0$ & $150.0 \pm 3.0$ \\
OD $(200 \mathrm{mg})$ & $162.2 \pm 4.8$ & $322.1 \pm 4.7$ & $111.7 \pm 5.2$ \\
\hline
\end{tabular}

(a)

\begin{tabular}{lccc}
\hline & Control & OD $(100 \mathrm{mg})$ & OD $(200 \mathrm{mg})$ \\
\hline $3 \mathrm{~mm} \leq x<5 \mathrm{~mm}$ & $22.4 \pm 12.0$ & $18.3 \pm 5.5$ & $19.5 \pm 2.6$ \\
$5 \mathrm{~mm} \leq x<10 \mathrm{~mm}$ & $8.9 \pm 1.9$ & $11.5 \pm 6.5$ & $10.4 \pm 3.5$ \\
$x \geq 10 \mathrm{~mm}$ & $6.2 \pm 4.8$ & $2.1 \pm 3.7$ & $1.7 \pm 4.2$ \\
\hline
\end{tabular}

(b)

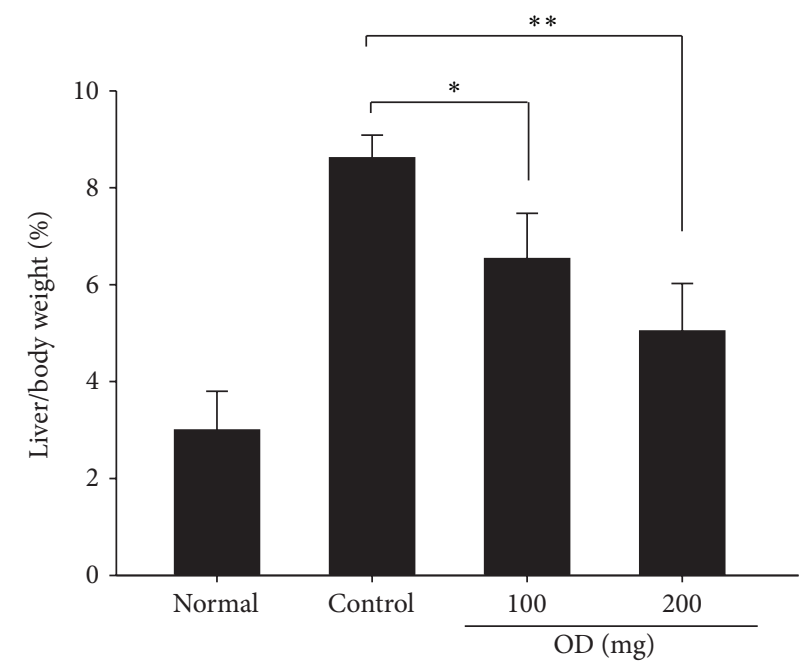

(c)

FIGURE 4: The analysis of level on serum, tumor number categorized by tumor size, and assessment of the liver/body weight 28 days after OD treatment. The blood samples were collected 28 days after OD treatment and the serum level of ALP, AST, and ALT was measured (a). Comparison of tumor number was categorized by tumor size 28 days after OD treatment (b). Also, liver/body weight ratio was measured 28 days after OD treatment. The serum levels of ALP, AST, and ALT in OD treated group were significantly decreased compared to the control group (a). Also, a larger tumor burden in OD treated group was observed less than control group (b). The liver/body weight ratio in rats after OD treatment (c). OD treated group significantly reduced the liver/body ratio compared to the control group. Columns, mean; bar, SD. ${ }^{*} P<0.05$ and ${ }^{* *} P<0.01$.
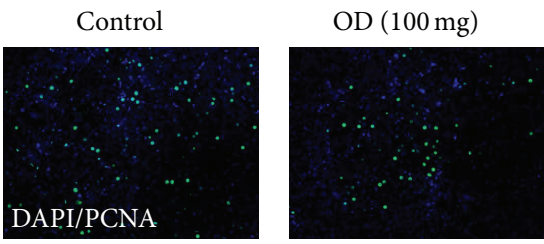

(a)

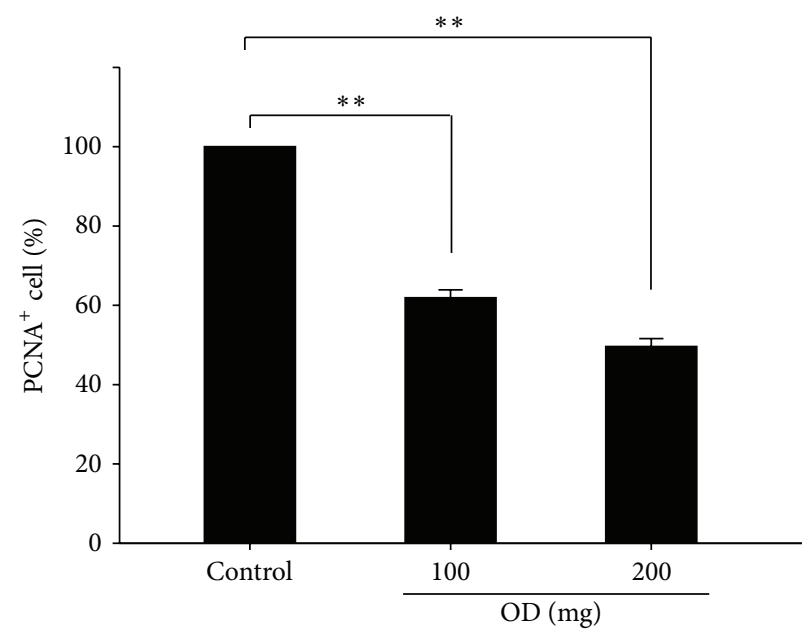

(b)

FIGURE 5: Inhibition of tumor cell proliferation by OD. Histological analysis was shown in PCNA-positive cells 28 days after OD treatment ((a) scale bars, $100 \mu \mathrm{m})$. Quantification of PCNA-positive cells in the tumor area (b). The proliferated cells were significantly decreased in the OD treated group (100 and $200 \mathrm{mg} / \mathrm{kg}$ ) compared to the control group. Columns, mean; bar, SD. ${ }^{* *} P<0.01$. 


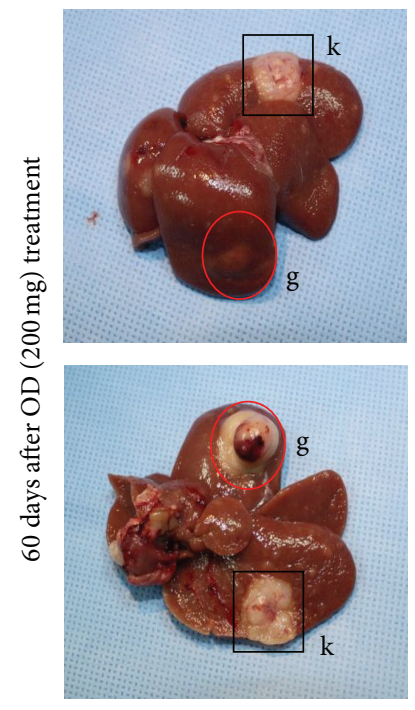

(a)

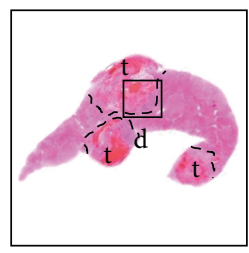

(c)

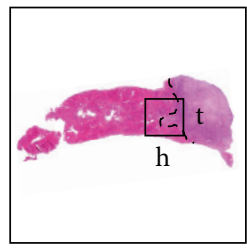

(g)

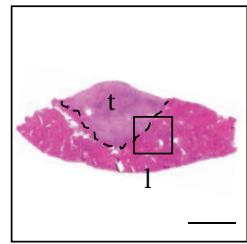

(k)

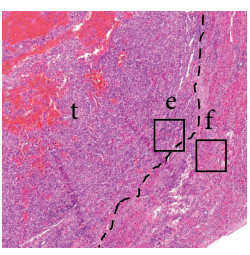

(d)

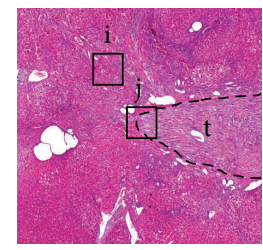

(h)

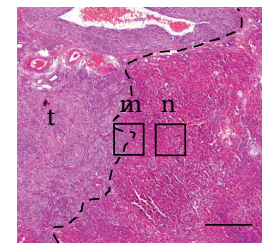

(l)

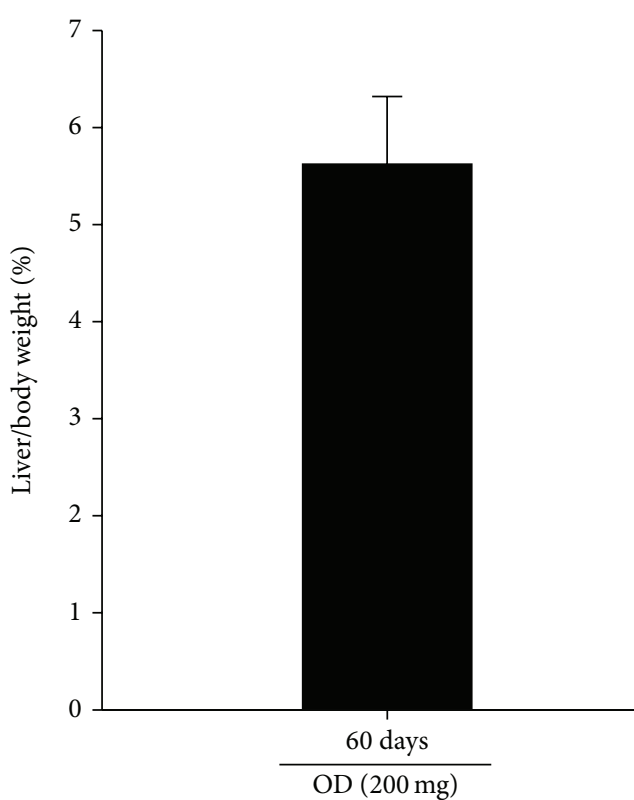

(b)

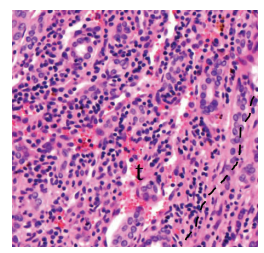

(e)

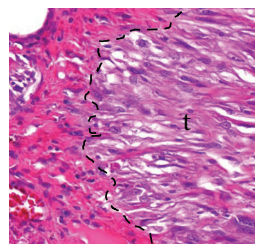

(i)

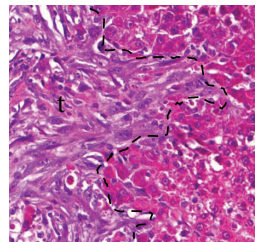

(m)

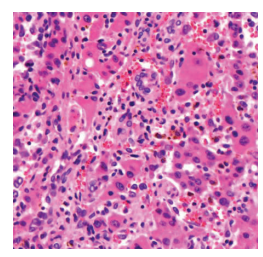

(f)

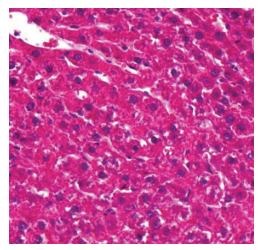

(j)

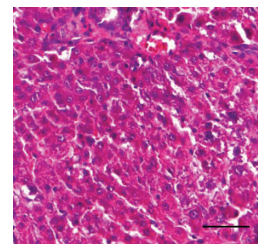

(n)

FIGURE 6: Liver morphology, liver/body ratio, and histopathology analysis 60 days after OD treatment. Liver/body weight ratio was measured after last OD treatment $(200 \mathrm{mg} / \mathrm{kg})$. Liver morphology was shown 60 days after OD treatment $(200 \mathrm{mg} / \mathrm{kg})$ and $\mathrm{H} \& \mathrm{E}$ staining was done. The large tumor observed in two sites (a). Except for tumor site, the rest of the liver tissue was similar to the normal liver morphology. OD $(200 \mathrm{mg} / \mathrm{kg})$ inhibits metastasis of tumor. Liver/body weight ratio in rats 60 days after OD treatment (b). The liver/body ratio after OD treatment $(200 \mathrm{mg} / \mathrm{kg})$ was not different between 28 days and 60 days (columns, mean; bar, SD). We show that tumor growth including the malignant nodules over $3 \mathrm{~mm}$ of a diameter, cirrhosis, and necrosis in tumor were significantly progressing in slides at 28 days in control group $((\mathrm{c})-(\mathrm{f})) .60$ days after OD treatment, we observed the boundary between the large tumor and normal liver cells $((\mathrm{g})-(\mathrm{f}),(\mathrm{k})-(\mathrm{m}))$. Also, no cirrhosis was shown and normal liver cells exist ((j), (n)). Scale bars ((a), (g), and (k)); $5 \mathrm{~mm}((\mathrm{~d}),(\mathrm{h})$, and (l)); $500 \mu \mathrm{m}((\mathrm{e})-(\mathrm{f}),(\mathrm{i})-(\mathrm{j})$, and $(\mathrm{m})-(\mathrm{n})) ; 50 \mu \mathrm{m}$. 
architecture (Figures 6(j) and 6(n)). These results suggest that OD $(200 \mathrm{mg} / \mathrm{kg})$ may inhibit the ability of cell proliferation and metastasis of tumor through enhanced hepatic function.

\section{Discussion}

Herbal medicine has been used in Asian countries and numerous studies have reported anticancer activity in vitro using a natural herb extract $[8,26]$. OD, a traditional Chinese and Korean medicinal plant, is known for its anticancer activities $[14,27,28]$ and is a valuable medicinal plant for its multipurpose uses. However, the mechanism of antitumor effect of OD is still largely unknown and has not been evaluated in chemically induced liver cancer. In this study, we investigated the anticancer effect of $\mathrm{OD}$ on chemically induced liver cancer model. Also, we studied the anticancer effect of OD such as apoptosis, cell proliferation, and migration of HCC cells including huh7 and hepG2 and elucidated possible mechanisms.

This study demonstrated that OD induced apoptosis by caspase-3 pathway in HCC cells. Also, OD suppressed proliferation of HCC cells compared to untreated control cells. These results were consistent with previous findings of anticancer effect in breast cancer cells [14]. Since HCC exhibits a high rate of metastasis, it would be of great importance to understand the migration receptor related gene expression that affects the process of HCC metastasis. We were able to demonstrate the effect of OD in inhibiting cell migration in HCC cells and reducing expression of migration related chemokine receptors such as CXCR1, CXCR2, and CXCR4. Both CXCR1 and CXCR2 are predominantly expressed within the immune system and bind with the highest affinity to the chemokine interleukin-8. CXCR1 and CXCR2 can mediate migration, invasion, and proliferation individually in cancer cells [29]. CXCR4, known as SDF-1 $\alpha$ receptor, plays a critical role in normal physiology as well as the pathology of many human diseases including cancer, inflammation, and autoimmune disease. Also, CXCR4 correlates with the histological grade and invasive capacity of tumor cells, as well as with tumor cell survival [30]. Our results suggest that OD has anticancer effect in caspase- 3 induced apoptosis, antiproliferation, and the inhibition of migration. However, we do not know the exact mechanism of OD as OD consists of numerous compounds. We hypothesized that the principal component in OD including OA and UA could have antitumor effects. The OA and UA are present in OD at high abundance and are pentacyclic triterpenoids that exist widely in plants [31,32]. OA and UA share similar chemical structure and the only difference of the two compounds is the position of one methyl group between $\mathrm{C} 19$ and C20. Our results show OA and UA inhibit cell proliferation of huh7 and hepG2. Also, OA and UA induce apoptosis involving mitochondrial pathway through caspase-3 activation and reduced expression of migration related receptors such as CXCR1, CXCR2, and CXCR4. Our results suggest that OA and UA presented in OD at high abundance can play critical roles of anticancer effects.

We investigated the anticancer effect of $\mathrm{OD}$ in in vivo model using the chemically induced liver cancer model.
We employed a DEN-induced cancer model which can establish HCC and liver cirrhosis simultaneously as the malignant tumor progresses from liver cirrhosis. This model may provide similarity between experimental and human HCC and be a better scheme for studying human HCC than the implanted HCC model with cirrhosis. The survival in vivo experiment revealed that $\mathrm{OD}$ enhances survival rate compared to the control group. Also, $30 \%$ of rats in OD treated group survived more than 50 days. ${ }^{18} \mathrm{~F}$-FDG can provide a useful indicator of tumor growth and metastasis because ${ }^{18} \mathrm{~F}$-FDG accumulates in tumor mass with increased glucose metabolism [33]. Also, ${ }^{18}$ F-FDG imaging can provide additional information including tumor development and secondary tumor growth. In a previous study, we have reported that we analyzed ${ }^{18}$ F-FDG imaging using the PET/CT time-dependently in DEN-induced liver cancer model. We analyzed ${ }^{18}$ F-FDG imaging at day 0 and day 28 . The uptake of ${ }^{18} \mathrm{~F}-\mathrm{FDG}$ of liver in OD treated group was lower in certain areas compared to the control group. Furthermore, OD can change hepatic function related factors including ALT, AST, and ALP on serum level in HCC model. Serum components such as ALT, AST, and ALP are markers of hepatic function and their increases in blood indicate liver damage by the cytotoxic effects of DEN $[34,35]$.

Our results show that OD may regulate glucose metabolism in tumors through enhancing hepatic function. Also, these results show similar pattern as reduction of the number categorized by tumor size, liver/body ratio, and cell proliferation in tumors. We investigated liver morphology, tumor number, and size of rats that survived more than 60 days after OD treatment. We observed the large tumor in two sites. Except for tumor site, the rest of the liver tissue was similar in morphology to normal liver, despite the chemically induced HCC model generating numerous tumors and cirrhosis in the liver (Figure 4(b)). This is an interesting finding suggesting that OD may have a role in enhancing the hepatic function and inhibiting the metastasis in chemically induced HCC model. In the pathology analysis, we observed normal hepatocellular architecture in the boundary around the tumor site. Also, the liver/body weight ratio in rats 28 days and 60 days after OD treatment was not different. These results suggest that OD inhibits the ability of cell proliferation and tumor metastasis through the regulation of hepatic function, glucose metabolism, and the migration related receptors.

In conclusion, this study demonstrated that OD treatment has anticancer effects in inhibiting the growth of HCC cell in chemically induced liver cancer model. Also, OD can regulate the hepatic function and glucose metabolism. Furthermore, our results have shown that OA and UA derived from OD extracts act as the bioactive components of the anticancer effects. Further studies are needed to identify the detailed mechanisms involved in the therapeutic effect of OD in vivo.

\section{Conflict of Interests}

The authors report no conflict of interests. 


\section{Authors' Contribution}

Yun-Young Sunwoo and Jin-Hee Lee equally contributed to this work as joint first authors.

\section{Acknowledgment}

The research was supported by the Incheon St. Mary's Hospital of Catholic University of Korea, through Institute of Catholic Integrative Medicine Foundation program.

\section{References}

[1] M. M. E. Taha, A. B. Abdul, R. Abdullah, T. A. T. Ibrahim, S. I. Abdelwahab, and S. Mohan, "Potential chemoprevention of diethylnitrosamine-initiated and 2-acetylaminofluorenepromoted hepatocarcinogenesis by zerumbone from the rhizomes of the subtropical ginger (Zingiber zerumbet)," ChemicoBiological Interactions, vol. 186, no. 3, pp. 295-305, 2010.

[2] A. Jemal, F. Bray, M. M. Center, J. Ferlay, E. Ward, and D. Forman, "Global cancer statistics," CA Cancer Journal for Clinicians, vol. 61, no. 2, pp. 69-90, 2011.

[3] S. S. Thorgeirsson and J. W. Grisham, "Molecular pathogenesis of human hepatocellular carcinoma," Nature Genetics, vol. 31, no. 4, pp. 339-346, 2002.

[4] M. A. Kern, K. Breuhahn, and P. Schirmacher, "Molecular pathogenesis of human hepatocellular carcinoma," Advances in Cancer Research, vol. 86, pp. 67-112, 2002.

[5] V. Moudgil, D. Redhu, S. Dhanda, and J. Singh, "A review of molecular mechanisms in the development of hepatocellular carcinoma by afatoxin and hepatitis B and C viruses," Journal of Environmental Pathology, Toxicology and Oncology, vol. 32, no. 2, pp. 165-175, 2013.

[6] M. Branda and J. R. Wands, "Signal transduction cascades and hepatitis B and C related hepatocellular carcinoma," Hepatology, vol. 43, no. 5, pp. 891-902, 2006.

[7] K. Hanajiri, H. Mitsui, T. Maruyama, N. Hashimoto, M. Sata, and M. Omata, "Echographic detection of diethylnitrosamineinduced liver tumors in rats and the effect of the intratumoral injection of an inhibitor of c-Jun N-terminal kinase," Journal of Gastroenterology and Hepatology (Australia), vol. 24, no. 5, pp. 866-871, 2009.

[8] K.-I. Park, H.-S. Park, S.-R. Kang et al., "Korean Scutellaria baicalensis water extract inhibits cell cycle G1/S transition by suppressing cyclin D1 expression and matrixmetalloproteinase-2 activity in human lung cancer cells," Journal of Ethnopharmacology, vol. 133, no. 2, pp. 634-641, 2011.

[9] Z. Xu, X. Chen, Q. Zhang, L. Chen, and Y. Wang, "Corydalis yanhusuo W.T. Wang extract inhibits MCF-7 cell proliferation by inducing cell cycle G2/M arrest," The American Journal of Chinese Medicine, vol. 39, no. 3, pp. 579-586, 2011.

[10] Y.-Q. Wang, S.-J. Zhang, H. Lu, B. Yang, L.-F. Ye, and R.-S. Zhang, "A $\mathrm{C}_{21}$-steroidal glycoside isolated from the roots of Cynanchum auriculatum induces cell cycle arrest and apoptosis in human gastric cancer sgc-7901 cells," Evidence-Based Complementary and Alternative Medicine, vol. 2013, Article ID 180839, 7 pages, 2013.

[11] L. Yang, X. Liu, Z. Lu et al., "Ursolic acid induces doxorubicinresistant HepG2 cell death via the release of apoptosis-inducing factor," Cancer Letters, vol. 298, no. 1, pp. 128-138, 2010.
[12] P. K. Wu, W. Chi Shing Tai, Z. T. Liang, Z. Z. Zhao, and W. L. W. Hsiao, "Oleanolic acid isolated from Oldenlandia diffusa exhibits a unique growth inhibitory effect against rastransformed fibroblasts," Life Sciences, vol. 85, no. 3-4, pp. 113121, 2009.

[13] M. Ganbold, J. Barker, R. Ma, L. Jones, and M. Carew, "Cytotoxicity and bioavailability studies on a decoction of Oldenlandia diffusa and its fractions separated by HPLC," Journal of Ethnopharmacology, vol. 131, no. 2, pp. 396-403, 2010.

[14] G. Gu, I. Barone, L. Gelsomino et al., "Oldenlandia diffusa extracts exert antiproliferative and apoptotic effects on human breast cancer cells through ER $\alpha /$ Sp1-mediated p53 activation," Journal of Cellular Physiology, vol. 227, no. 10, pp. 3363-3372, 2012.

[15] K.-A. Kim, J.-S. Lee, H.-J. Park et al., "Inhibition of cytochrome P450 activities by oleanolic acid and ursolic acid in human liver microsomes," Life Sciences, vol. 74, no. 22, pp. 2769-2779, 2004.

[16] S.-L. Yan, C.-Y. Huang, S.-T. Wu, and M.-C. Yin, "Oleanolic acid and ursolic acid induce apoptosis in four human liver cancer cell lines," Toxicology in Vitro, vol. 24, no. 3, pp. 842-848, 2010.

[17] J. Li, W.-J. Guo, and Q.-Y. Yang, "Effects of ursolic acid and oleanolic acid on human colon carcinoma cell line HCT15," World Journal of Gastroenterology, vol. 8, no. 3, pp. 493-495, 2002.

[18] Y. Lu, M. Zhu, W. Chen et al., "Oleanolic acid induces apoptosis of MKN28 cells via AKT and JNK signaling pathways," Pharmaceutical Biology, vol. 52, no. 6, pp. 789-795, 2014.

[19] X. Wang, H. Bai, X. Zhang et al., "Inhibitory effect of oleanolic acid on hepatocellular carcinoma via ERK-p53-mediated cell cycle arrest and mitochondrial-dependent apoptosis," Carcinogenesis, vol. 34, no. 6, pp. 1323-1330, 2013.

[20] M.-H. Shyu, T.-C. Kao, and G.-C. Yen, "Oleanolic acid and ursolic acid induce apoptosis in HuH7 human hepatocellular carcinoma cells through a mitochondrial-dependent pathway and downregulation of XIAP," Journal of Agricultural and Food Chemistry, vol. 58, no. 10, pp. 6110-6118, 2010.

[21] W. Chang, S. I. Park, S.-Y. Jun et al., "Therapeutic potential of autologous mesenchymal stem cells derived from synovial fluid in patients with degenerative arthritis," Animal Cells and Systems, vol. 17, no. 5, pp. 315-324, 2013.

[22] T. Y. Lee, K.-T. Kim, and S.-Y. Han, "Expression of ErbB receptor proteins and TGF-alpha during diethylnitrosamine-induced hepatocarcinogenesis in the rat liver," The Korean Journal of Hepatology, vol. 13, no. 1, pp. 70-80, 2007.

[23] E. Schiffer, C. Housset, W. Cacheux et al., "Gefitinib, an EGFR inhibitor, prevents hepatocellular carcinoma development in the rat liver with cirrhosis," Hepatology, vol. 41, no. 2, pp. 307314, 2005.

[24] Y. Y. Sunwoo, S. I. Park, Y. A. Chung et al., "A pilot study for the neuroprotective effect of Gongjin-dan on transient middle cerebral artery occlusion-induced ischemic rat brain," Evidence-Based Complementary and Alternative Medicine, vol. 2012, Article ID 682720, 11 pages, 2012.

[25] S. I. Park, J. H. Lee, H. J. Ham et al., "Evaluation of 2$\left[{ }^{18} \mathrm{~F}\right]$-fluoro-2-deoxy-d-glucose positron emission tomography/computed tomography in rat model on hepatocellular carcinoma with liver cirrhosis," Bio-Medical Materials and Engineering, article 1339, 2015.

[26] D.-H. Lee, K.-I. Park, H.-S. Park et al., "Flavonoids isolated from Korea Citrus aurantium L. induce G2/M phase arrest and apoptosis in human gastric cancer AGS cells," Evidence-Based 
Complementary and Alternative Medicine, vol. 2012, Article ID 515901, 11 pages, 2012.

[27] S. Willimott, J. Barker, L. A. Jones, and E. I. Opara, "Apoptotic effect of Oldenlandia diffusa on the leukaemic cell line HL60 and human lymphocytes," Journal of Ethnopharmacology, vol. 114, no. 3, pp. 290-299, 2007.

[28] S. Gupta, D. Zhang, J. Yi, and J. Shao, "Anticancer activities of Oldenlandia diffusa," Journal of Herbal Pharmacotherapy, vol. 4, no. 1, pp. 21-33, 2004.

[29] S. A. Khurram, L. Bingle, B. M. McCabe, P. M. Farthing, and S. A. Whawell, "The chemokine receptors CXCR1 and CXCR2 regulate oral cancer cell behaviour," Journal of Oral Pathology \& Medicine, vol. 43, no. 9, pp. 667-674, 2014.

[30] S. A. Rempel, S. Dudas, S. Ge, and J. A. Gutiérrez, "Identification and localization of the cytokine SDF1 and its receptor, CXC chemokine receptor 4 , to regions of necrosis and angiogenesis in human glioblastoma," Clinical Cancer Research, vol. 6, no. 1, pp. 102-111, 2000.

[31] B. B. Aggarwal and S. Shishodia, "Molecular targets of dietary agents for prevention and therapy of cancer," Biochemical Pharmacology, vol. 71, no. 10, pp. 1397-1421, 2006.

[32] J. Liu, "Pharmacology of oleanolic acid and ursolic acid," Journal of Ethnopharmacology, vol. 49, no. 2, pp. 57-68, 1995.

[33] C. Messa, Y. Choi, C. K. Hoh et al., "Quantification of glucose utilization in liver metastases: parametric imaging of FDG uptake with PET,' Journal of Computer Assisted Tomography, vol. 16, no. 5, pp. 684-689, 1992.

[34] C. Gupta, A. Vikram, D. N. Tripathi, P. Ramarao, and G. B. Jena, "Antioxidant and antimutagenic effect of quercetin against DEN induced hepatotoxicity in rat," Phytotherapy Research, vol. 24, no. 1, pp. 119-128, 2010.

[35] S. Santhosh, T. K. Sini, R. Anandan, and P. T. Mathew, "Hepatoprotective activity of chitosan against isoniazid and rifampicininduced toxicity in experimental rats," European Journal of Pharmacology, vol. 572, no. 1, pp. 69-73, 2007. 


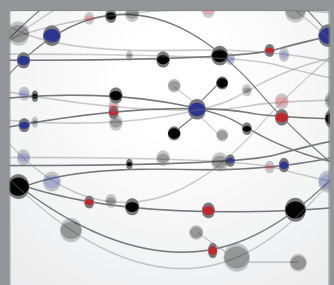

The Scientific World Journal
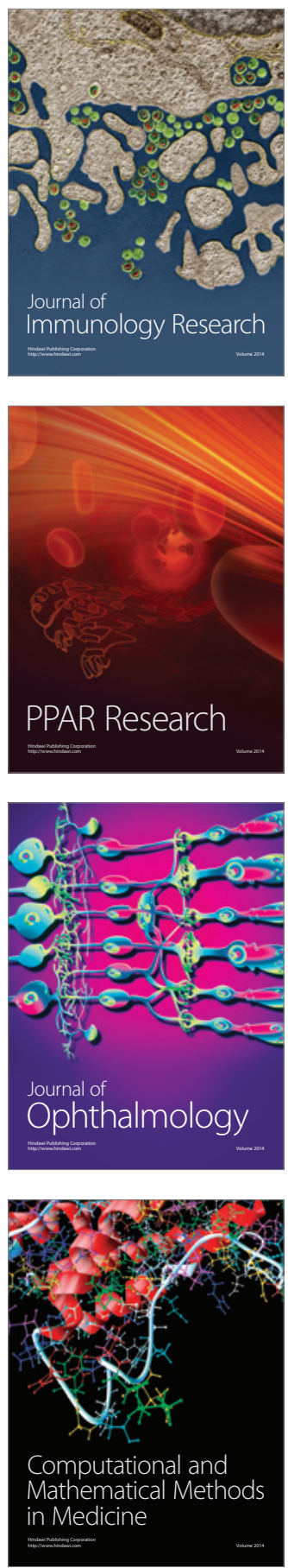

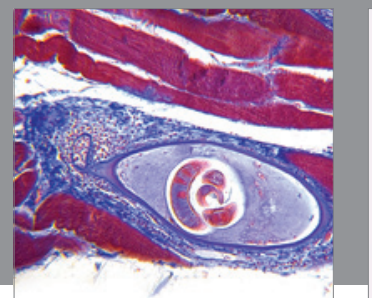

Gastroenterology

Research and Practice
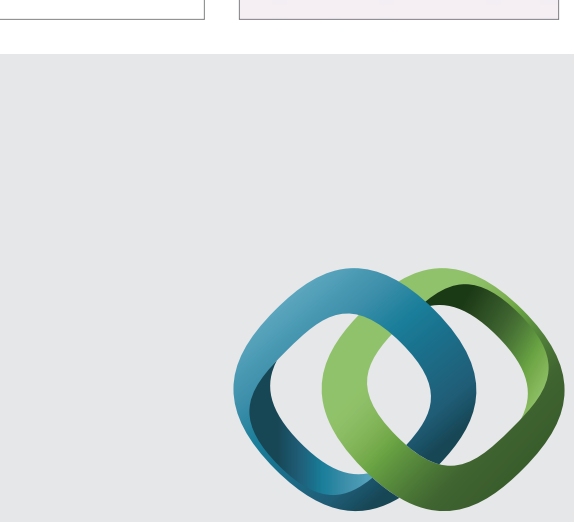

\section{Hindawi}

Submit your manuscripts at

http://www.hindawi.com
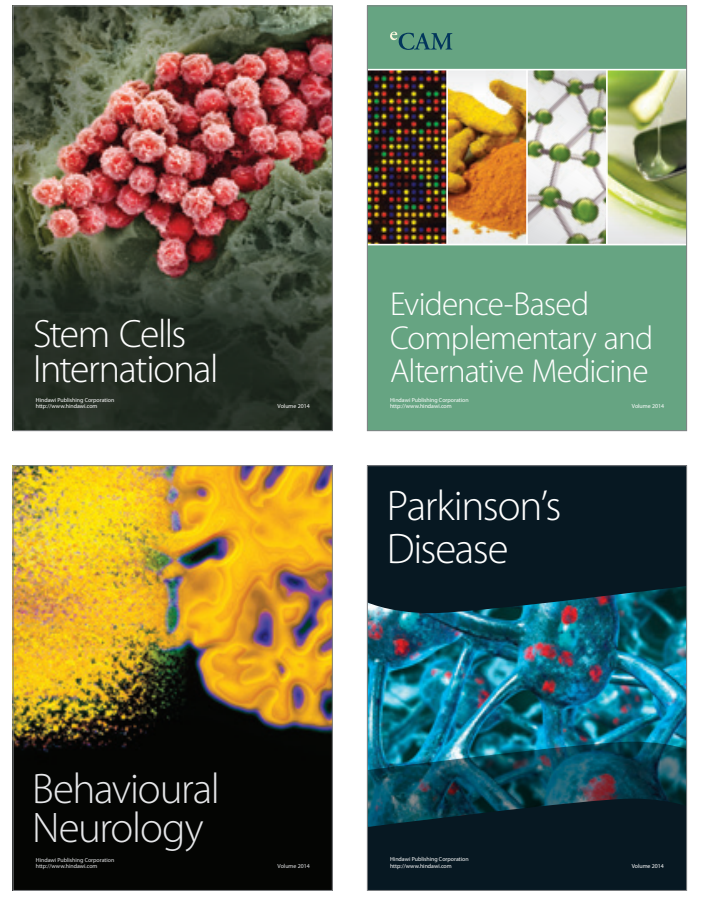
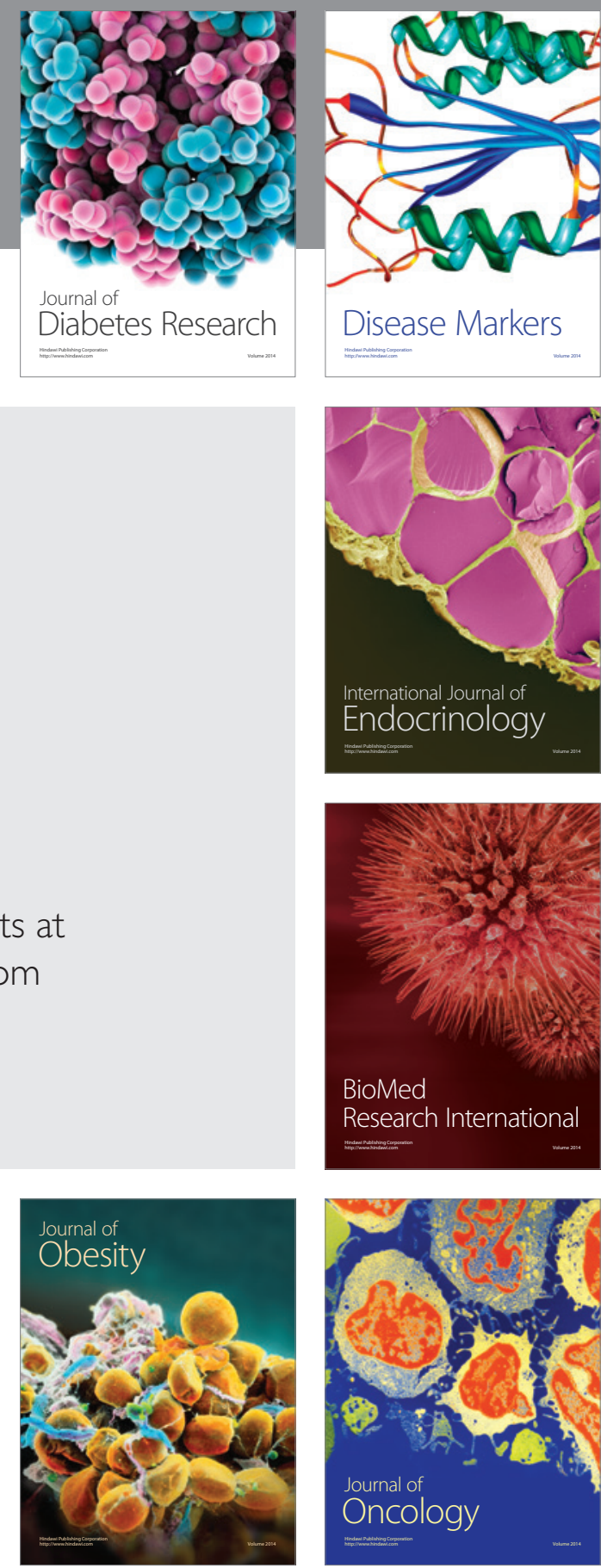

Disease Markers
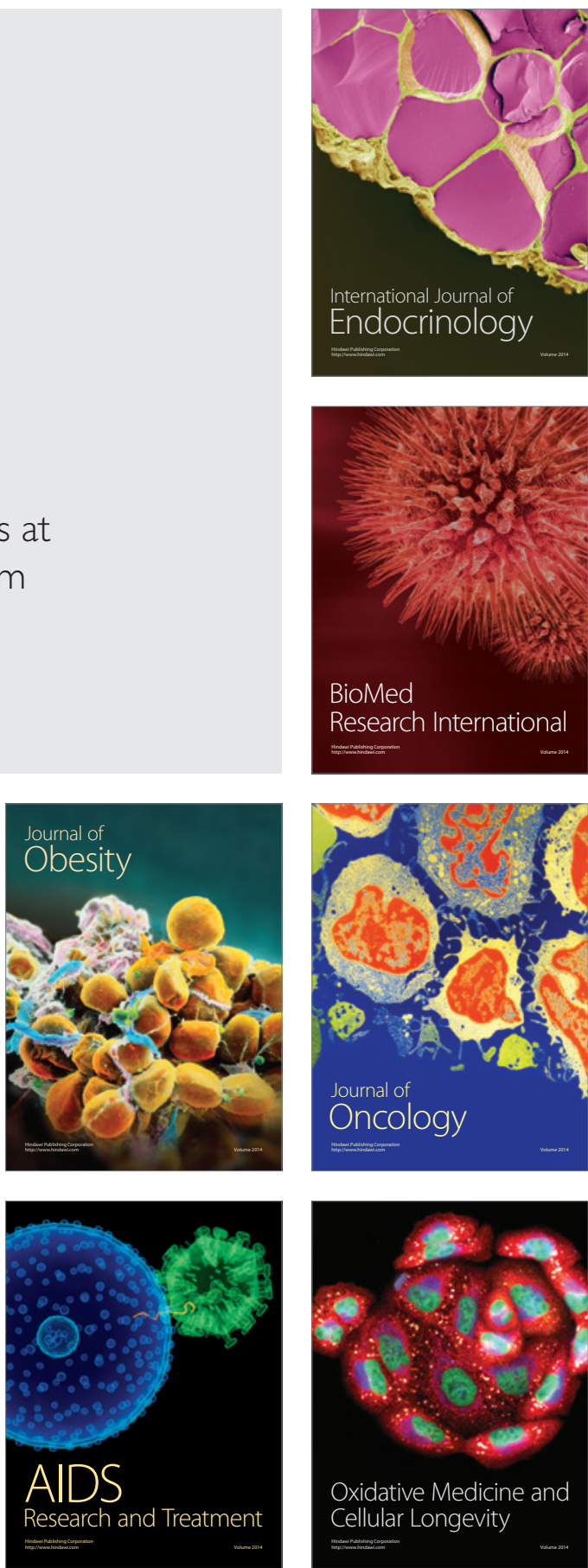